BULLETIN (New Series) OF THE

AMERICAN MATHEMATICAL SOCIETY

Volume 48, Number 4, October 2011, Pages 495-496

S 0273-0979(2011)01352-0

Article electronically published on June 20, 2011

\title{
INTRODUCTORY COMMMENTS
}

\author{
SUSAN FRIEDLANDER
}

Donald Saari, who was my predecessor as Chief Editor of the Bulletin of the American Mathematical Society, used the excuse of the new millennium to devote an issue in 2000 to review the development of mathematics in the early 20th century by reprinting a number of fascinating articles that appeared in the Bulletin in this period. Don hoped that these early articles "will encourage readers to enter the dusty, far reaches of mathematics libraries to thumb through old Bulletin issues". Undoubtedly, the tactile experience of holding and turning the pages of old books is a delightful one and cannot be replaced by reading the computer screen. However, modern technology has its conveniences and, thanks to a recent AMS project, all the issues of the Bulletin from its inception in 1891 to the present are freely available to everyone on the web. Now that we are a decade into the 21st century, I invited some of the current Bulletin Editors to browse the back issues electronically and select an article that was particularly meaningful to them. This present issue contains the choices of six of the Editors together with a short commentary by these Editors explaining why they made their selection.

Of course, six articles can cover only a small taste of the stunning advances in mathematics that have taken place in the past generation. However, these seminal articles reflect the vitality of diverse areas of mathematics that were stimulated by work of excellent mathematicians in the last half of the 20th century. It is interesting, but in no sense surprising, that each Editor chose an article that was particularly important to them when they were themselves beginning their mathematical careers. With one exception, all the choices are from the period 1977 to 1984. This was indeed a fine decade for mathematics. The time period also indicates, for those who like to make guesses, the mathematical age of many of the Bulletin Editors.

Andrew Ranicki chose a pair short announcements that appeared in 1958, one by Raoul Bott and John Milnor and the second by Frank Adams. He notes that the "story" of the Hopf invariant is once again hot news with the recent results of Hopkins, Hill, and Ravenel. We anticipate an article on this breakthrough result in a future issue of the Bulletin. Daniel Freed also chose an article by Bott on "Morse theory, old and new". This was published in 1982, thus we have two snapshots from the impressive career of Raoul Bott. We continue the spotlight on algebraic topology with the selected reprinting from Mathematical Reviews. In May of 2011 the Abel Prize was awarded to John Milnor. To celebrate this award, we chose to reprint six reviews of Milnor's seminal papers in the decade 1956-1966. We note that most of these excellent reviews were written by Frank Adams (over his career Adams published 397 Math Reviews!) 
On "nontopological" topics the choices were the following:

- Gregory Lawler choose Edward Nelson's article "Internal set theory: a new approach to nonstandard analysis" which appeared in 1977.

- David Benson chose Richard Stanley's article "Invariants of finite groups and their applications to combinatorics" which appeared in 1979.

- Bryna Kra chose Hillel Furstenberg's article "Poincaré recurrence and number theory" published in 1981.

- Edward Frenkel chose Steven Gelbart's article "An elementary introduction to the Langland's Program" published in 1984.

I hope you will enjoy reading these personal selections of the Editors who are always seeking new Bulletin articles that are "future classics" in the spirit of the articles reprinted in this issue. Perhaps you will like to visit www.ams.org, click on the Bulletin icon, and browse the back issues to find your own favorite.

Department of Mathematics, University of Southern California, Los Angeles, CalIFORNIA 90089

E-mail address: susanfri@usc.edu 